\title{
Social Media Handbuch
}

\author{
Andreas Hoffelder
}

Rezension von Daniel Michelis; Thomas Schildhauer (Hrsg.) (2010). Social Media Handbuch - Theorien, Methoden, Modelle. Nomos.

Das Social-Media-Handbuch ist ein Versuch, bei der rasanten Entwicklung der sozialen Medien in den letzten Jahren einen Überblick zu liefern; aufgrund der Fülle neuer Studien und Ergebnisse, die in dem Bereich beinahe täglich publiziert werden, ein ambitioniertes Vorhaben. Die Fülle an Informationen, die aus den sozialen Medien auf jeden einzelnen einwirken, ist kaum mehr zu verarbeiten. Das vorliegende Buch möchte nun einen Schritt zurück treten und das Geschehen von einer übergeordneten/wissenschaftlichen Perspektive betrachten. Langfristige Trends und grundsätzliche Zusammenhänge sollen so besser erkannt werden.

Der erste Teil des Social-Media-Handbuchs ist in erster Linie eine Bestandsaufnahme ausgewählter Theorien, Modelle und Methoden von führenden Autoren auf dem Gebiet, welche sich als Leitfaden für die Entwicklung eigener Antworten, Lösungen und Strategien anbietet. Hier werden unter anderem Lawrence Lessings „The Future of Ideas“ oder James Surowieckis „Die Weisheit der Vielen“ inhaltlich dargestellt sowie Kernaussagen herausgearbeitet und Praxistipps abgeleitet. Jeder der insgesamt 13 vorgestellten Publikationen wird hier ein eigenes Kapitel gewidmet. Anfangs werden die einzelnen Social Media - Elemente des Web 2.0, also etwa Youtube oder Facebook, mit ihren Funktionen dargestellt. Von der Sprache im Web 2.0 über das Entstehen von Trends bis hin zu Erklärungen zu den konkreten Nutzungsmöglichkeiten verschiedener Portale werden die Inhalte theoretisch abstrakt erläutert.

Der praktische zweiteTeil bietet Fallbeispiele, in welchen besagte Theorien, Methoden und Modelle angewandt werden.
Die Inhalte des ersten Teils werden exemplarisch zur Lösung realer Probleme in der Praxis angewandt. So erläutern die beiden Herausgeber des Buches Daniel Michelis und Thomas Schildhauer zusammen mit Sascha Gysel im ersten Kapitel des zweiten Teils die strategischen und operativen Erfolgsfaktoren der sozialen Medien des Web 2.0 am Beispiel der FacebookKampagne des WWF.

Das Handbuch unterscheidet sich vom Großteil der bisherigen Veröffentlichungen von selbsternannten Social Media Päpsten vor allem dadurch, dass es trotz großer Praxisnähe zumindest in großen Teilen wissenschaftlichen Ansprüchen genügt. Der aktuelle Stand der Forschung wird komprimiert und verständlich dargelegt. Ein weiterer Vorteil des Buches ist, dass es nicht lediglich die aktuellen Trends zusammenfasst, sondern allgemeine Funktionsweisen einer Social Media erläutert und somit der Gefahr entgegenwirkt, schnell veraltet zu sein. Der gute systematische Aufbau erleichtert die methodische Entwicklung eigener Anwendungen und Lösungen. So werden sowohl im ersten als auch im zweiten Teil Modelle, Begriffe, Kernsätze oder Autoreninfos in eingerahmten Kästen hervorgehoben, was das Buch auch als Nachschlagewerk eignet,

Dem Leser bietet das Buch einen guten Einstieg in das sehr aktuelle Feld der Social Media. Durch den Fokus auf die praktischen Aspekte des Themas kann das Buch eine wichtige Hilfestellung beim Aufbau einer eigenen Social Media sein. Zudem stellt es einen guten Einstieg für Themenneulinge und eine gute Hilfe für Fortgeschrittene dar, da man sich mit diesem Werk viel Recherche und Nachschlagen ersparen kann.

Andreas Hoffelder Hertie School of Governance hoffelder@hertie-school.org

\section{Media Events in a Global Age}

\author{
Sandro Gianella and Petter Malvik
}

Book Review of Couldry, N. / Hepp, A. \& Krotz, F. (2010). Media Events in a Global Age. Routledge.

In their seminal book Media Events: The Live Broadcasting of History, Daniel Dayan and Elihu Katz came up with the highly influential notion of the media event. Bringing together communication research on media studies and semiotics-influenced media and culture studies, the two authors defined a media event as "high holidays of mass communication".
According to Dayan and Katz these "communication holidays" are characterized by, among other things being: preplanned, celebrative, having an integrative function on the national level and being broadcasted exclusively on TV.

Given today's media landscape, this definition is arguably somewhat outdated. With the rise of online news, TV-broadcasting has lost some of its prominence, while news at the same time have become increasingly global, making the original focus on national audiences seem somewhat passé. Given 